Ann. Biol. anim. Bioch. Biophys., I97I, 11 (3), 455-469.

\title{
INFLUENCE D'UN APPORT DE DL-MÉTHIONINE A DES VACHES AU DÉBUT DE LA LACTATION SUR LA PRODUCTION LAITIÈRE ET LA COMPOSITION DU SANG
}

\author{
B. REMOND, C. CHAMPREDON*, C. DECAEN, R. PION* et M. JOURNET
}

avec la collaboration technique de Renée Lefaivre, J. Lefaivre et B. Marquis

Station de Recherches sur l'Élevage des Ruminants,

*Station d'Étude des Métabolismes,

Centre de Recherches zootechniques et vétérinaires, I. N. R. A., 63 - Saint-Genès-Champanelle

\section{RÉSUMÉ}

A un lot de I I vaches laitières nous avons distribué $50 \mathrm{~g}$ de $\mathrm{DL}$-méthionine par jour et par animal (lot méthionine); nous avons comparé ses productions à celles d'un autre lot de vaches (lot témoin), traité de la même façon, mais qui ne recevait pas de méthionine. La distribution de méthionine a commencé 2 semaines avant le vêlage et s'est terminée à la $5^{\mathbf{e}}$ semaine de la lactation.

Les quantités d'aliments ingérées ont été les mêmes dans les 2 lots (tabl. 2 ); il en est de même des quantités de lait et de matières azotées produites, et de la teneur du lait en matières azotées (tabl. 2 et fig. I). En revanche, le taux butyreux et la quantité de matières grasses sécrétée dans le lait ont été plus importants dans le lot méthionine que dans le lot témoin $(\mathrm{P}<0,05)$. Le poids vif des vaches a diminué après le vêlage dans le lot méthionine, mais a augmenté dans le lot témoin. Dans le lot méthionine, comparativement au lot témoin, la composition des acides gras du lait n'a pas été significativement différente bien que le pourcentage des acides gras longs a eu tendance à être plus élevé (tabl. 3) ; par ailleurs, la concentration des corps cétoniques dans le sang a été plus élevée $(P<0,10)$, ainsi que celle des acides gras libres dans le plasma (de façon non significative).

A la $5^{e}$ semaine de la lactation la concentration des acides aminés libres totaux du sang a été plus faible dans le lot méthionine que dans le lot témoin, ce qui a été principalement dû̀ à une diminution de la concentration des acides aminés indispensables (tabl. 5) ; la concentration des acides aminés du cycle de l'urée a aussi été plus faible. A la différence des autres acides aminés indispensables, les concentrations de la méthionine et de la lysine n'ont pas été plus faibles dans le lot méthionine que dans le lot témoin, et la concentration de l'histidine a même été plus élevée.

Il semble que la méthionine ait pu agir au niveau digestif en stimulant la croissance des microorganismes du rumen et en particulier des protozoaires, au niveau métabolique en accroissant la mobilisation des lipides corporels et aussi peut-être au niveau de la mamelle en accroissant l'activité de synthèse. 


\section{INTRODUCTION}

Etudiant la composition des différentes classes de lipides des lipoprotéines sériques chez des vaches normales et acétonémiques, et se fondant sur les rôles connus de la méthionine dans le métabolisme lipidique, McCarThy et al. (1968) ont émis 1'hypothèse qu'une carence en cet acide aminé pouvait être un facteur important de l'apparition de l'acétonémie chez les bovins. A l'appui de cette hypothèse, ces auteurs ont montré qu'un apport de méthionine ou d'un analogue hydroxylé (MHA) permettait une amélioration rapide de l'état de santé de vaches acétonémiques et une augmentation importante de leur production laitière.

L'action favorable de la supplémentation du régime en MHA sur la quantité de lait produite et plus encore sur le taux butyreux a été mise en évidence avec des vaches en bon état de santé au début de la lactation (GRIEL et al., I968; PolAN et al., I970 $a$; PatTon et al., I970 $b$ ). Cependant, BuRgos et OLson (I970) qui distribuaient des quantités de MHA semblables à celles des auteurs précédemment cités à des vaches qui, semble-t-il, n'étaient pas au début de la lactation, n'ont trouvé aucun effet favorable. Enfin, CHANDLER et al., I97o, n'ont pas enregistré de variation de production laitière chez des vaches auxquelles étaient infusés $20 \mathrm{~g}$ de MHA par jour pendant 6 jours. Des expériences effectuées aux Etats-Unis, on peut conclure que la distribution de MHA a un effet favorable sur la quantité de lait produite et le taux butyreux quand il est distribué au début de la lactation à raison de 25 à $45 \mathrm{~g}$ par jour environ; distribué en plus faible quantité, il n'a pas d'action, tandis qu'en quantité plus importante, il a une action défavorable sur la quantité de lait produite à cause, semble-t-il, de son effet dépressif sur les quantités d'aliments ingérées.

Les expériences réalisées avec la méthionine n'ont pas été plus nombreuses. WiLLiams et al. (I970) n'ont pas trouvé d'effet sur la production laitière en distribuant I2 $\mathrm{g}$ par jour de DL-méthionine protégée; de même, BRODERIck et al. (I970) pour des doses variant de 5 à $45 \mathrm{~g}$ par jour. Les infusions intraveineuses de L-méthionine ne se sont pas révélées plus efficaces (Teichman et al. Ig69, ChandLER et al., 1970). Mais contrairement aux expériences qui ont mis en évidence l'intérêt de la supplémentation en MHA (cf. ci-avant), aucune de celles réalisées avec la méthionine n'a été effectuée avec des vaches qui étaient au début de la lactation.

Les modes d'action de la méthionine et du MHA sont encore mal connus. Au niveau de la digestion, le MHA accroîtrait la digestibilité de la matière sèche, de la cellulose brute et, quand l'aliment concentré est broyé, celle des matières azotées (PoLAN et al., I970 b). Il augmenterait aussi la concentration des protozoaires dans le jus de rumen (PATron et al., I97o $c$ ), tandis que in vitro, la méthionine stimulerait la synthèse des lipides (PATTON et al., I968 et I970 a).

L,e MHA ajouté à la ration pourrait aussi avoir une action directe sur le métabolisme de 1'animal, d'autant que PoLAN et al. (I970 a) et GRIEL et al. (rg68) ont noté que sa distribution augmentait la concentration de la méthionine dans le sang. Avec des vaches au début de la lactation, Polan et al. (I970 a) ont constaté que la distribution de MHA entraînait une modification de la composition des lipides plasmatiques ; les acides gras libres et les triglycérides avaient disparu des $\alpha$-lipoprotéines, et les triglycérides, des $\beta$-lipoprotéines. CHANDLER et al. (I970) ont observé 
une augmentation significative de la concentration des lipides totaux, des triglycérides et des acides gras libres du plasma. Parron et al. (I97o b) ont aussi observé une augmentation de la concentration des lipides totaux plasmatiques.

Des quelques études réalisées, il ressort que si 1'action du MHA sur la sécrétion du lait et des matières grasses au début de la lactation semble établie, en revanche celle de la méthionine ne l'est pas. Quant à leurs modes d'action, ils restent encore à préciser.

La France est un des principaux pays producteurs de méthionine ; aussi avonsnous utilisé la méthionine à la place du MHA. Dans une expérience effectuée avec des vaches fortes productrices au début de la lactation, nous avons voulu observer l'influence de la distribution journalière de 50 grammes de DL-méthionine sur la quantité de lait produite et sa composition, le poids vif des animaux et la concentration de certains constituants du sang.

\section{MATÉRIEL, ET MÉTHODES}

\section{Schéma expérimental et alimentation}

Pendant l'hiver 1969-1970, 22 vaches (I4 Frisonnes, 6 Holstein, I Normande et I Frisonne $\times$ Montbéliarde) ont été réparties environ 4 semaines avant le vêlage en 2 lots destinés l'un à recevoir une supplémentation en méthionine (lot méthionine) et l'autre non (lot témoin). La constitution des 2 lots a été effectuée en tenant compte de la race, du numéro de la lactation, de la date du vêlage, et de la quantité de lait produite et de sa composition pendant la lactation précédente. Au cours de celle-là, le numéro de la lactation, la quantité de lait produite en 305 jours et les teneurs du lait en matières grasses et en matières azotées ont été respectivement de $\mathrm{I}, 7-44 \mathrm{I} 7 \mathrm{~kg}-$ $37,4 \mathrm{p}$. Iooo et $33,9 \mathrm{p}$. I ooo pour le lot méthionine, et I,5 $-4300 \mathrm{~kg}-36,7 \mathrm{p}$. Iooo et $33,9 \mathrm{p}$. I ooo pour le lot témoin.

TABLEAU I

Composition chimique des aliments et coefficient d'utilisation digestive apparent
de la matière organique des fourrages

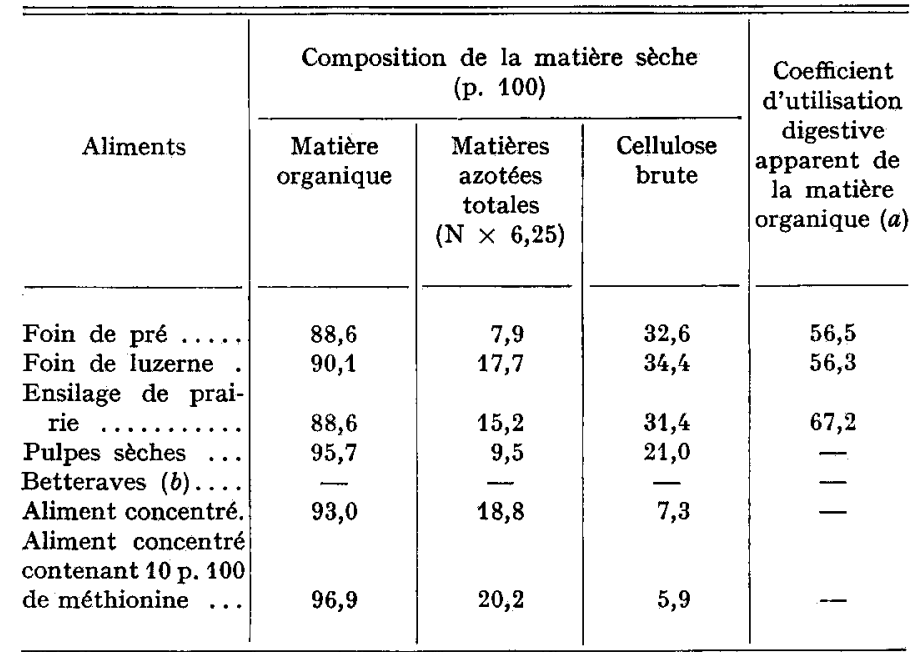

a : mesurée avec 4 moutons

b : la composition n'a pas été mesurée 
La composition chimique des aliments est rapportée dans le tableau I. Pour tous les animaux la ration de base était constituée de foins (de graminées et de luzerne) et d'ensilage d'herbe, offerts à volonté, ainsi que de betteraves riches en matière sèche ou de pulpes. Un aliment concentré était donné 2 fois par jour : avant le vêlage, en faible quantité (respectivement $2,75 \mathrm{~kg}$ et $3,05 \mathrm{~kg}$ pendant les semaines-2 et -I), et après le vêlage en quantité croissante de façon que les besoins théoriques des animaux, déduits de leur production laitière pendant les $4^{\mathrm{e}}, 5^{\mathrm{e}}$ et $6^{\mathrm{e}}$ jours de la lactation (DEcaEN et al., I970), soient couverts vers la fin du premier mois de la lactation. L'aliment concentré était composé de $55 \mathrm{p}$. Ioo d'orge, $20 \mathrm{p}$. Ioo d'avoine, ro p. Ioo de tourteau d'arachide, ro p. roo de tourteau de colza et 5 p. Ioo d'un mélange de minéraux. En substitution à cet aliment concentré, les vaches du lot méthionine ont reçu chaque jour, en 2 distributions, de 2 semaines avant à 5 semaines après le vêlage, chacune $500 \mathrm{~g}$ d'un aliment concentré préparé par l'A. E. C., contenant Io p. Ioo de DL-méthionine.

Dans la suite de l'exposé, on a appelé période expérimentale, celle qui débutait la $2^{\mathbf{e}}$ semaine avant le vêlage et se terminait la $5^{\mathbf{e}}$ semaine de la lactation, et période post-expérimentale, les 6 et $7^{\mathbf{e}}$ semaines de la lactation.

\section{Mesures}

Voici, avec leur fréquence, les différentes mesures individuelles qui ont été effectuées : quantités d'aliments ingérées, chaque jour ; quantité de lait produite, à chaque traite ; teneur du lait en matières grasses (méthode Gerber), chaque jour ; teneur du lait en matières azotées (méthode au noir amido II B), une fois par semaine; poids vif des animaux, une fois par semaine.

La composition des acides gras du lait a été mesurée par chromatographie en phase gazeuse des esters méthyliques et butyliques (DECAEN et ADDA, I966), avec un chromatographe muni de

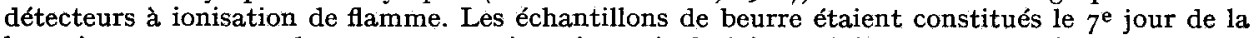
lactation, et au cours des $3^{\mathrm{e}}$ et $7^{\mathrm{e}}$ semaines, à partir du lait produit en une journée.

Les mêmes jours, du sang était prélevé au niveau de la veine jugulaire, dans un flacon hépariné, avant la distribution des aliments du matin. Dans le sang entier, on a mesuré sur toutes les vaches, la concentration du glucose par la méthode à la glucose-oxydase (HuGGETT et NIXoN, 1957), et, sur 4 vaches de chaque lot, celle des corps cétoniques (Procos, 196I). Dans le plasma, on a mesuré sur toutes les vaches, la concentration des acides gras libres (Dole et MeINERTz, 1960), et celle des lipides totaux (Folch et al., r957) sur 4 vaches de chaque lot pour le prélève-

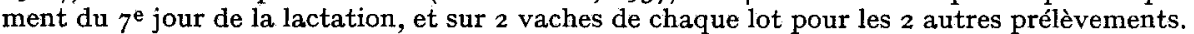

D'autres prélèvements de sang ont été faits au niveau de la veine jugulaire, sur 7 vaches de chaque lot, 3 heures après la distribution des aliments du matin, au cours de la $5^{\mathrm{e}}$ semaine après le vêlage pour le lot témoin, et des $5^{\mathrm{e}}$ et $7^{\mathrm{e}}$ semaines pour le lot méthionine. Les échantillons de sang correspondant au même lot et à la même semaine ont été mélangés en quantité égale. Dans l'échantillon moyen ainsi obtenu, les acides aminés libres ont été extraits par épuisements successifs à l'éthanol à 84 p. IOO (PAwLAK et Pron, I968) et dosés par colorimétrie après séparation par chromatographie sur des colonnes de résines échangeuses d'ions, au moyen d'un analyseur automatique. riance.

L'analyse des résultats zootechniques a été effectuée statistiquement par analyse de cova-

\section{RÉSULTATS}

Les résultats de caractère zootechnique (quantités d'aliments ingérées, quantité de lait produite et composition du lait, poids vif et bilan énergétique) des 2 lots d'animaux sont rassemblés d'une part dans le tableau 2 qui présente les moyennes obtenues pendant les 5 premières semaines de la lactation, période sur laquelle a porté l'analyse statistique des résultats, et d'autre part dans la figure I qui illustre l'évolution de quelques variables de 2 semaines avant à 7 semaines après le vêlage.

\section{Quantités d'aliments ingérées}

Les quantités ingérées de chaque aliment (exprimées en matière sèche) ont été en moyenne les mêmes pour les 2 lots d'animaux au cours de l'expérience. Le tableau 2 
TABLEAU 2

Quantités d'aliments ingérées, production laitière et bilan énergétique pendant les 5 premières semaines de la lactation

(moyennes journalières)

\begin{tabular}{|c|c|c|c|}
\hline Moyenne par vache & $\begin{array}{l}\text { Lot } \\
\text { méthionine } \\
\text { A }\end{array}$ & $\begin{array}{l}\text { Lot } \\
\text { témoin } \\
\qquad B\end{array}$ & $\begin{array}{l}\text { Analyse de } \\
\text { covariance; seuil } \\
\text { de signification }\end{array}$ \\
\hline 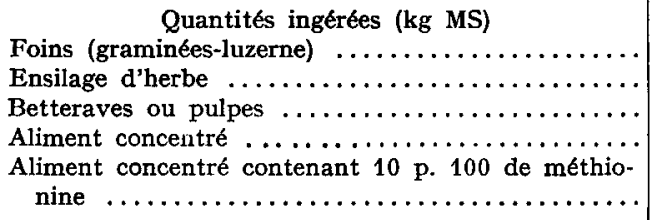 & $\begin{array}{l}3,92 \\
2,80 \\
3,06 \\
5,72 \\
0,43\end{array}$ & $\begin{array}{l}4,03 \\
2,89 \\
3,20 \\
6,12 \\
0\end{array}$ & $\begin{array}{l}\text { NS } \\
\text { NS } \\
\text { NS }\end{array}$ \\
\hline 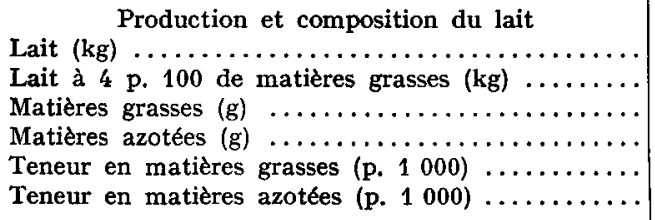 & $\begin{array}{c}23,6 \\
23,5 \\
934 \\
837 \\
39,7 \\
35,6\end{array}$ & $\begin{array}{l}23,5 \\
22,2 \\
852 \\
817 \\
\because 6,4 \\
\mathbf{3 4 , 9}\end{array}$ & $\begin{array}{l}\text { NS } \\
\text { NS } \\
5 \text { p. } 100 \\
\text { NS } \\
5 \text { p. } 100 \\
\text { NS }\end{array}$ \\
\hline 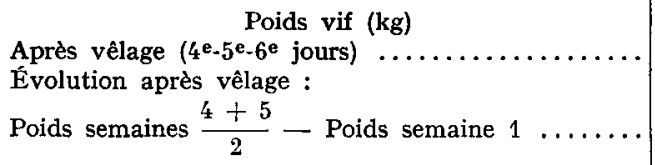 & $\begin{array}{r}581 \\
-\quad 11,6\end{array}$ & 584 & $\begin{array}{l}\text { NS } \\
5 \text { p. } 100\end{array}$ \\
\hline $\begin{array}{l}\text { Bilan énergétique (UF) } \\
\text { (Apports-besoins) } \\
\text { Après vêlage (moyenne }+1 \text { à }+5 \text { ) } \ldots \ldots \ldots \ldots\end{array}$ & 1,0 & 0,4 & \\
\hline
\end{tabular}

présente les quantités d'aliments ingérées au cours des 5 premières semaines de la lactation. Pendant les 2 semaines qui ont précédé le vêlage les quantités ingérées de ration de base (somme de tous les aliments, excepté 1'aliment concentré) et de ration totale ont été respectivement de $9,2 \mathrm{~kg}$ et $12,2 \mathrm{~kg}$ pour le lot méthionine, et de $8,5 \mathrm{~kg}$ et II,3 $\mathrm{kg}$ pour le lot témoin ; pendant les $6^{\mathrm{e}}$ et $7^{\mathrm{e}}$ semaines de la lactation (période post-expérimentale), elles ont respectivement été de II,o $\mathrm{kg}$ et I $8,3 \mathrm{~kg}$ pour le lot méthionine et de Ir, $8 \mathrm{~kg}$ et $\mathrm{I} 8,5 \mathrm{~kg}$ pour le lot témoin. Au cours de la période expérimentale (de 2 semaines avant le vêlage à 5 semaines après), les animaux du lot méthionine ont ingéré par jour $50 \mathrm{~g}$ de méthionine libre de plus que les animaux du lot témoin; ils en ont encore ingéré en moyenne Io $\mathrm{g}$ de plus par jour pendant la $6^{\mathbf{e}}$ semaine de la lactation.

\section{Quantités de lait produites et composition du lait}

Les productions laitières initiales (moyenne des $4^{\mathrm{e}}, 5^{\mathrm{e}}$ et $6^{\mathrm{e}}$ jours de la lactation) ont été sensiblement identiques et respectivement de 20,9 et 2 I,3 $\mathrm{kg}$ pour les lots méthionine et témoin. Ces derniers avaient donc un potentiel de production compa- 
rable car, d'après JOURNET (résultats non publiés), la quantité de lait produite jusqu'aux environs du $5^{\mathrm{e}}$ jour de la lactation est encore peu influencée par le niveau d'alimentation. La quantité maximale de lait produite (moyenne journalière d'une semaine de mesures) a été de $26, \mathrm{I} \mathrm{kg}$ et $25,3 \mathrm{~kg}$ respectivement pour les lots méthionine et témoin, et a eu lieu au cours des $5^{\mathrm{e}}$ et $4^{\mathrm{e}}$ semaines de lactation. L'augmentation de la quantité de lait produite a donc été différente entre les 2 lots : elle a été plus importante, comparativement à la production moyenne des $4^{\mathrm{e}}, 5^{\mathrm{e}}$ et $6^{\mathrm{e}}$ jours, ou à celle de la $2^{\mathrm{e}}$ semaine, et a duré plus longtemps dans le lot méthionine que dans le lot témoin. En moyenne, pour les 5 premières semaines, les quantités journalières de lait produites n'ont pas été significativement différentes entre les 2 lots (respectivement 23,6 et $23,5 \mathrm{~kg}$ pour les lots méthionine et témoin). Il en est de même pour le lait à $4 \mathrm{p}$. xoo de matières grasses, malgré une différence de $\mathrm{r}, 2 \mathrm{~kg}$ en faveur du lot méthionine. Pendant les 2 semaines post-expérimentales, la production laitière des vaches du lot méthionine a continué à être supérieure à celle des animaux du lot témoin, de la même quantité que pendant la $5^{\mathrm{e}}$ semaine de lactation : de $\mathrm{r}, 6 \mathrm{~kg}$ de lait et de $\mathrm{I}, 8 \mathrm{~kg}$ de lait à $4 \mathrm{p}$. Ioo de matières grasses.
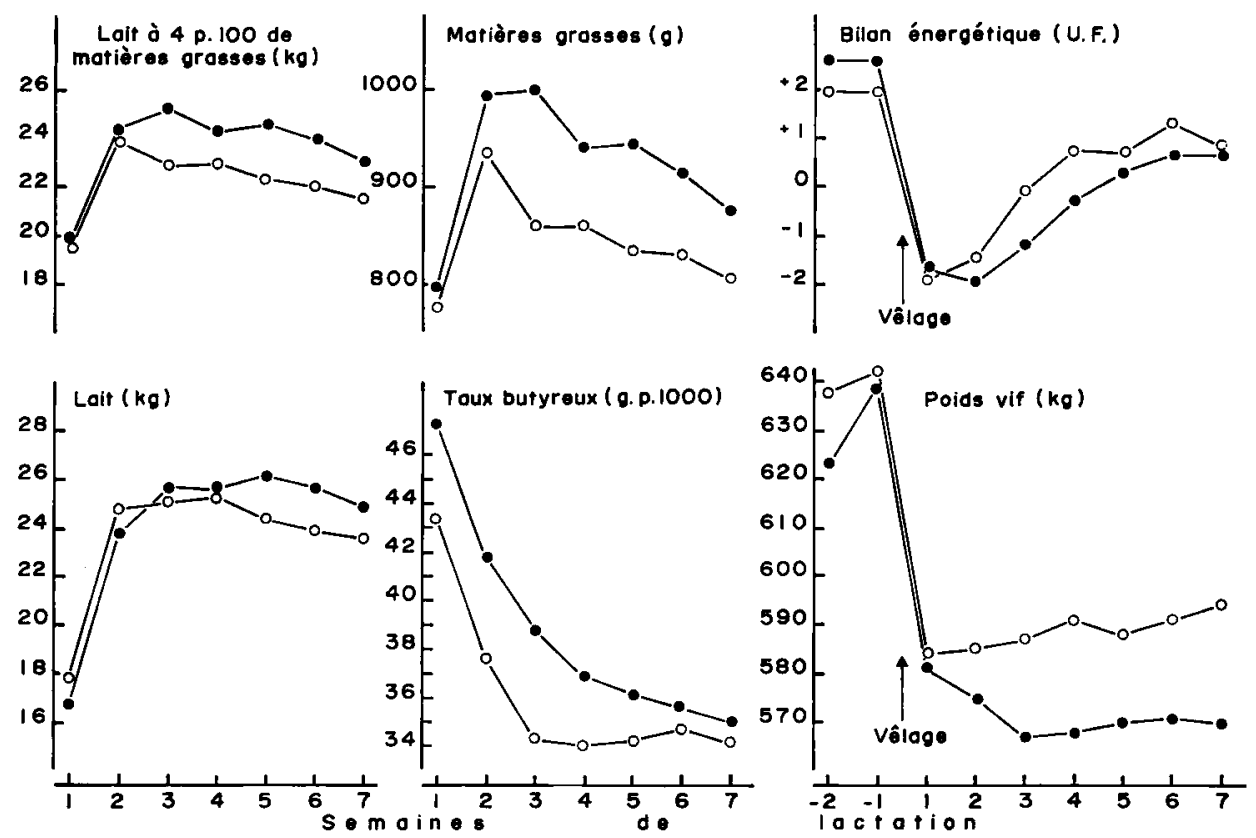

FIG. I. - Évolution au début de la lactation des quantités de lait et de matières grasses produites, du taux butyreux, du bilan energétique et du poids vif

$\because$ Lot méthionine

Pendant toute l'expérience, le taux butyreux des animaux du lot méthionine a été supérieur à celui des animaux du lot témoin, de $3,3 \mathrm{~g} \mathrm{p}$. I ooo en moyenne pour les 5 premières semaines de lactation $(P<0,05)$. Dans le lot méthionine, il a toujours diminué, rapidement pendant les 4 premières semaines puis plus lentement ensuite, tandis que dans le lot témoin il n'a diminué que pendant les 3 premières 
semaines de la lactation (parallèlement à celui du lot méthionine), puis n'a plus évolué ensuite. Il en résulte qu'à partir de la $3^{\mathrm{e}}$ semaine de la lactation, la différence entre les taux butyreux des 2 lots a diminué d'une semaine à l'autre.

Les quantités moyennes de matières grasses sécrétées au cours des 5 premières semaines ont été plus élevées $(\mathrm{P}<0,05)$ pour le lot méthionine $(934 \mathrm{~g} / \mathrm{j})$ que pour le lot témoin $(852 \mathrm{~g} / \mathrm{j})$.

La teneur moyenne du lait en matières azotées n'a pas été significativement plus élevée pour le lot méthionine (différence de $0,7 \mathrm{~g} \mathrm{p}$. I 0oo). Les quantités de matières azotées produites n'ont pas été significativement différentes en tre les 2 lots

\section{Poids vif}

Le poids vif moyen des animaux du lot témoin a augmenté dès le début de la lactation, pendant les 7 semaines de l'expérience, tandis que dans le lot méthionine, il a diminué jusqu'à la semaine 3 (de $\mathrm{I} 4 \mathrm{~kg}$ ) puis a peu changé jusqu'à la semaine 7 . Entre le vêlage (juste après) et la moyenne des semaines $4+5$, le poids vif individuel des animaux a diminué d'en moyenne II, $6 \mathrm{~kg}$ dans le lot méthionine, mais a augmenté de 5,6 kg dans le lot témoin ; il a diminué pour Io des II vaches dans le lot méthionine et a augmenté pour 7 des II vaches dans le lot témoin. La variation de poids vif entre la semaine $I$ et la moyenne des semaines $4+5$ a été significativement différente entre les 2 lots $(P<0,05)$.

\section{Bilan énergétique}

Le bilan énergétique des 2 lots d'animaux a été calculé à partir des quantités d'aliments ingérées et de leur valeur énergétique pour les apports, des recommandations de FREDERICKSEN pour les besoins d'entretien (UF pour l'entretien $=\mathrm{I}, 5+$ poids vif (en centaine de $\mathrm{kg}$ )/2), et de celles de LEROY (I949) pour les besoins de production laitière $(0,38 \mathrm{UF}$ par $\mathrm{kg}$ de lait à $4 \mathrm{p}$. Ioo de matières grasses). Les valeurs énergétiques des foins et de l'ensilage ont été déduites de la digestibilité de leur matière organique mesurée avec des moutons, par la formule de BREIREM (I954).

Le bilan énergétique, négatif au début de la lactation, est devenu positif au cours des semaines 5 et 4 , respectivement pour les lots méthionine et témoin. Pendant les 2 premières semaines de la lactation le déficit énergétique a été à peu près identique pour les 2 lots d'animaux ( $\mathrm{I}, 8 \mathrm{UF}$ ). Pour les 5 premières semaines, le bilan énergétique des 2 lots d'animaux a été négatif, mais plus fortement (de o,6 UF par animal) dans le lot méthionine que dans le lot témoin.

\section{Composition des acides gras du lait}

I a composition des acides gras du lait est présentée dans le tableau 3. Pour les 2 lots d'animaux, les proportions relatives des acides palmitique + palmitoléique a eu tendance à augmenter avec le stade de lactation, et celle des acides gras longs $\left(\mathrm{C}_{18}+\mathrm{C}_{18: 1}\right)$ à diminuer. $\mathrm{L}$ a proportion des acides courts $\left(\mathrm{C}_{4} \mathrm{à}_{12}\right)$ a augmenté dans le lot méthionine, mais n'a pas varié dans le lot témoin. Les variations que nous avons observées sont beaucoup plus faibles que celles qu'avaient observées DECAEN et ADDA (rg66) avec des vaches qui étaient au même stade de lactation. Les 
TABLEAU 3

Composition des acides gras sécrétés dans le lait

(en p. Ioo d'esters méthyliques)

\begin{tabular}{|c|c|c|c|c|c|c|}
\hline \multirow{2}{*}{$\frac{\text { Lot }}{\text { Semaine de lactation }}$} & \multicolumn{3}{|c|}{ Méthionine } & \multicolumn{3}{|c|}{ Témoin } \\
\hline & 1 & 3 & 7 & 1 & 3 & 7 \\
\hline $\mathrm{C}_{4}$ & 4,58 & 5,18 & 4,51 & 5,26 & 4,49 & 4,20 \\
\hline$C_{6}$ & 2,77 & 3,12 & 2,99 & 3,19 & 2,83 & 3,05 \\
\hline $\mathrm{C}_{8}$ & 1,50 & 1,68 & 1,72 & 1,77 & 1,48 & 1,58 \\
\hline $\mathrm{C}_{10}$ & 3,14 & 3,94 & 4,00 & 3,66 & 3,30 & 3,81 \\
\hline $\mathrm{C}_{11}$ & 0,23 & 0,38 & 0,45 & 0,24 & 0,31 & 0,39 \\
\hline $\mathrm{C}_{12}$ & 4,09 & 4,83 & 4,84 & 4,36 & 4,71 & 5,07 \\
\hline $\mathrm{C}_{14}$ & 10,71 & 12,04 & 13,00 & 11,92 & 12,51 & 13,02 \\
\hline$C_{14: 1}$ & 1,31 & 1,41 & 1,77 & 1,20 & 1,64 & 1,81 \\
\hline$C_{15}$ & 1,12 & 1,21 & 1,59 & 1,22 & 1,48 & 1,66 \\
\hline$C_{15: 1}$ & 0,15 & 0,14 & 0,15 & 0,19 & 0,21 & 0,20 \\
\hline $\mathrm{C}_{8}$ & 29,81 & 31,28 & 32,88 & 29,11 & 32,41 & 34,70 \\
\hline$C_{16: 1}^{0}$ & 3,51 & 3,12 & 3,18 & 2,86 & 2,97 & 2,93 \\
\hline $\mathrm{C}_{7}$ & 0,88 & 0,84 & 0,67 & 0,75 & 0,67 & 0,76 \\
\hline$C_{17: 1}$ & 0,44 & 0,42 & 0,33 & 0,37 & 0,26 & 0,34 \\
\hline $\mathrm{C}_{18}$ & 9,96 & 8,84 & 7,84 & 9,80 & 8,74 & 7,48 \\
\hline$C_{18: 1}$ & 22,06 & 18,32 & 17,01 & 19,85 & 17,86 & 15,79 \\
\hline $\mathrm{C}_{18: 2}$ & 2,14 & 1,74 & 1,87 & 2,33 & 2,02 & 1,78 \\
\hline $\mathrm{C}_{18: 3}$ & 0,88 & 0,64 & 0,58 & 1,11 & 0,77 & 0,79 \\
\hline $\mathrm{C}_{20}$ & 0,68 & 0,73 & 0,55 & 0,68 & 0,72 & 0,54 \\
\hline
\end{tabular}

proportions des 3 classes d'acides gras n'ont pas été statistiquement différentes entre les 2 lots, quelle que soit la semaine de mesures considérée $\left(\mathrm{I}^{\mathrm{re}}, 3^{\mathbf{e}}\right.$ ou $\left.7^{\mathrm{e}}\right)$, mais la proportion des acides gras longs a eu tendance à être plus élevée dans le lot méthionine que dans le lot témoin.

Les rapports $\frac{\mathrm{C}_{16: 0}}{\mathrm{C}_{16: 1}}$ et $\frac{\mathrm{C}_{18: 0}}{\mathrm{C}_{18: 1}}$ qui donnent une idée de l'activité des désaturases de la glande mammaire, n'ont pas été significativement différents entre les 2 lots.

Les acides gras de chaque classe $\left(\mathrm{C}_{4}\right.$ à $\left.\mathrm{C}_{12} ; \mathrm{C}_{18}+\mathrm{C}_{16: 1} ; \mathrm{C}_{18}+\mathrm{C}_{18: 1}\right)$ ont été sécrétés en plus grande quantité par le lot méthionine que par le lot témoin, sauf les acides gras courts à la I re semaine expérimentale. Les acides gras longs ont toujours contribué, plus que les autres classes d'acides gras, à la plus grande quantité de matières grasses sécrétée par les animaux du lot méthionine.

\section{Teneurs du sang en certains constituants}

Glucose et corps cétoniques (tabl. 4.).

La concentration du glucose sanguin a eu tendance à augmenter avec l'avancement du stade de lactation, mais elle n'a pas été statistiqutement différente entre les 2 lots. La concentration des corps cétoniques n'a pas varié avec la semaine de lactation mais elle a été plus élevée dans le lot méthionine que dans le lot témoin 
TABLEAU 4

Concentration du glucose et des corps cétoniques dans le sang, et des lipides totaux et des acides gras libres dans le plasma

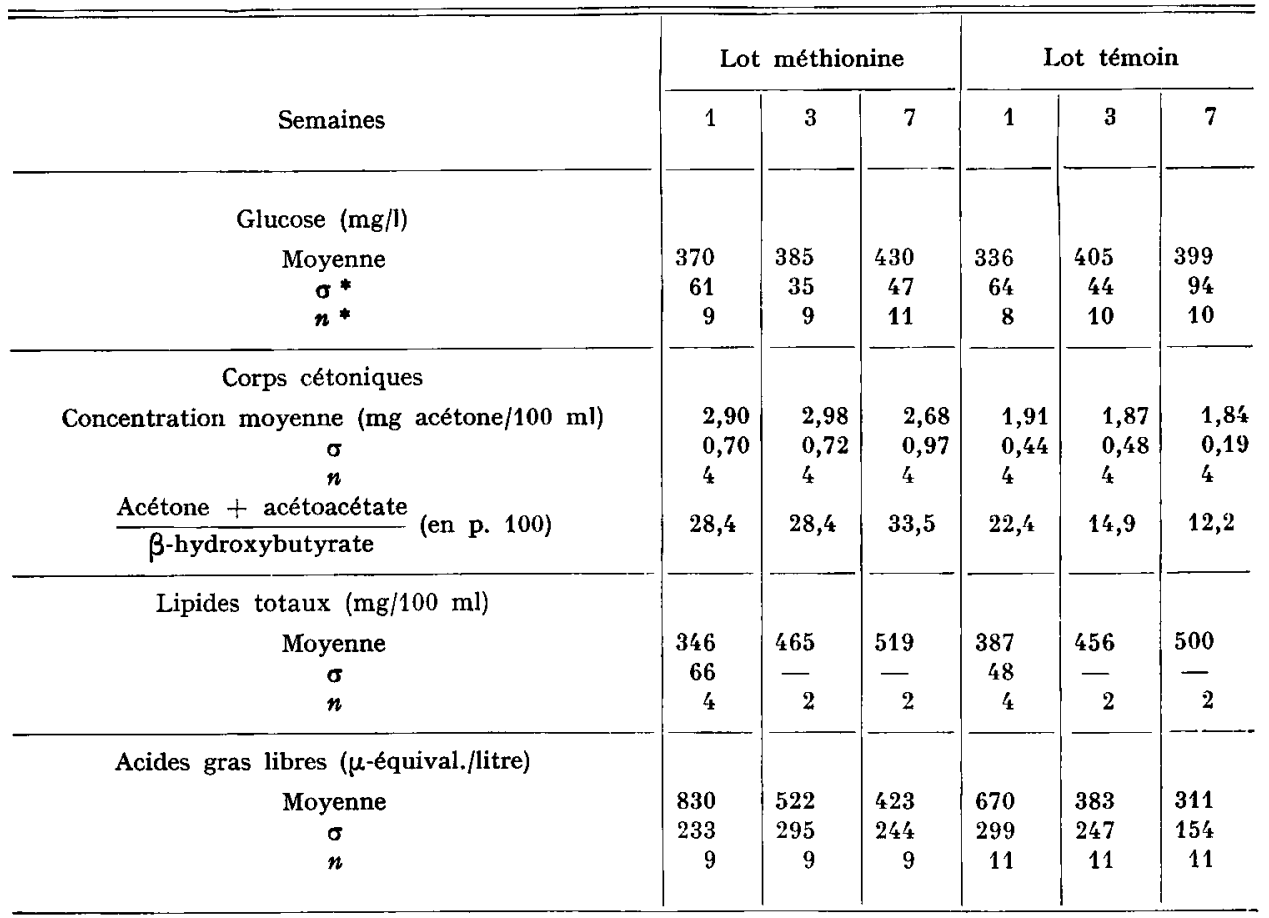

* $\sigma=$ écart-type

$n=$ nombre d'échantillons.

$(\mathrm{P}<0$,Io pour les semaines I et 3$)$. Pour chacune des 3 semaines de mesures $\left(\mathrm{I}^{\mathrm{re}}\right.$, $3^{\mathrm{e}}$, et $7^{\mathrm{e}}$ semaine), le rapport $\frac{\text { acétone }+ \text { acétoacétate }}{\beta \text {-hydroxybutyrate }}$ a été plus important dans $1_{\mathrm{e}}$ lot méthionine que dans le lot témoin. La différence n'est significative $(\mathrm{P}<0,05)$ que si on compare les valeurs obtenues à la $3^{\mathrm{e}}$ semaine de la lactation (4 données par lot) ou pendant toute l'expérience (I2 données par lot).

Lipides totaux et acides gras libres (tab1. 4).

La concentration des lipides totaux dans le plasma a augmenté dans les 2 lots avec l'avancement de la lactation mais, pour chacune des 3 semaines de prélèvements, n'a pas été différente entre les 2 lots. La concentration des acides gras libres dans le plasma a diminué dans les 2 lots entre les semaines I et $3(\mathrm{P}<0,0 \mathrm{r})$ et 3 et 7 (de façon non significative). Pour chacune des 3 semaines de prélèvements, il n'y a pas eu de différence significative entre les lots méthionine et témoin, bien que la concentration moyenne des acides gras libres ait été plus élevée de $30 \mathrm{p}$. Ioo pour le lot méthionine que pour le lot témoin. 
Acides aminés libres (tabl. 5).

La présence dans la ration des animaux du lot méthionine d'une quantité importante de méthionine ne semble pas entraîner d'augmentation de la teneur en méthionine du sang, puisque les concentrations de cet acide aminé dans les échantillons prélevés chez les animaux des deux lots 5 semaines après le début de la lactation sont voisines et relativement faibles; elle a toutefois pour conséquence une série de modifications des concentrations des autres acides aminés : la somme des teneurs de

TABLEAU 5

Concentration (mg/roo $\mathrm{g}$ de sang) des acides aminés libres dans le sang

\begin{tabular}{|c|c|c|c|}
\hline Lots & Témoin & \multicolumn{2}{|c|}{ Méthionine } \\
\hline Semaine de la lactation & $\mathbf{5}$ & 5 & 7 \\
\hline Nombre de vaches & 7 & 7 & 7 \\
\hline Acide aspartique $\ldots \ldots$. & 1,84 & 2,07 & 1,93 \\
\hline Thréonine $\ldots \ldots \ldots \ldots \ldots \ldots \ldots \ldots \ldots \ldots \ldots \ldots \ldots \ldots$ & 1,24 & 1,06 & 1,15 \\
\hline Sérine.$\ldots \ldots \ldots \ldots \ldots \ldots \ldots$ & 1,04 & 0,97 & 1,03 \\
\hline Glutamine + asparagine..... & 2,73 & 2,46 & 2,57 \\
\hline Acide glutamique $\ldots \ldots \ldots \ldots$ & 1,02 & 0,88 & 0,98 \\
\hline 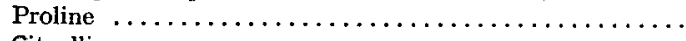 & 1,43 & 1,39 & 1,28 \\
\hline Citrulline $\quad \ldots \ldots \ldots \ldots \ldots \ldots \ldots \ldots \ldots \ldots \ldots \ldots \ldots \ldots$ & 1,46 & 1,04 & 1,41 \\
\hline Glycine $\ldots \ldots \ldots \ldots \ldots \ldots \ldots \ldots \ldots \ldots \ldots \ldots \ldots \ldots$ & 3,01 & 3,08 & 3,15 \\
\hline Alanine $\ldots \ldots \ldots \ldots \ldots \ldots \ldots \ldots \ldots \ldots \ldots \ldots \ldots \ldots \ldots \ldots \ldots$ & 2,01 & 1,65 & 2,03 \\
\hline 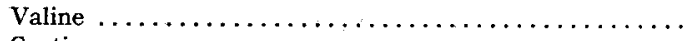 & 3,53 & 2,61 & 3,32 \\
\hline Cystine $\ldots \ldots \ldots \ldots \ldots \ldots \ldots \ldots \ldots \ldots \ldots \ldots \ldots \ldots \ldots \ldots \ldots \ldots$ & - & - & - \\
\hline Méthionine $\ldots \ldots \ldots \ldots \ldots \ldots \ldots \ldots \ldots \ldots \ldots \ldots \ldots \ldots$ & 0,50 & 0,46 & 0,44 \\
\hline Isoleucine $\ldots \ldots \ldots \ldots, \ldots, \ldots, \ldots, \ldots, \ldots, \ldots$ & 1,72 & 1,37 & 1,73 \\
\hline Leucine & 2,40 & 1,90 & 2,39 \\
\hline Tyrosine & 1,32 & 1,11 & 1,33 \\
\hline Phénylalanine..$\ldots \ldots \ldots \ldots \ldots \ldots$ & 1,08 & 0,80 & 1,03 \\
\hline Ornithine $\ldots \ldots \ldots \ldots \ldots \ldots \ldots \ldots \ldots \ldots \ldots \ldots \ldots \ldots \ldots \ldots \ldots$ & 0,97 & 0,90 & 0,99 \\
\hline 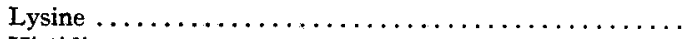 & 1,56 & 1,60 & 1,86 \\
\hline 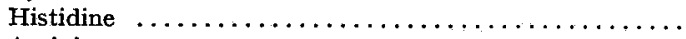 & 1,05 & 1,38 & 1,53 \\
\hline Arginine $\ldots \ldots \ldots \ldots \ldots \ldots \ldots \ldots \ldots$ & 0,71 & 0,65 & 0,84 \\
\hline Somme des & & & \\
\hline Acides aminés $\ldots \ldots \ldots \ldots \ldots \ldots \ldots \ldots \ldots \ldots \ldots$ & 30,62 & 27,41 & 31,01 \\
\hline Acides aminés indispensables et semi-indispensables ... & 15,11 & 12,96 & 15,62 \\
\hline Acides aminés non indispensables $\ldots \ldots \ldots \ldots \ldots \ldots$ & 15,51 & 14,45 & 15,38 \\
\hline Acides aminés du cycle de l'urée & 3,14 & 2,59 & 3,24 \\
\hline
\end{tabular}

l'ensemble des composés dosés est légèrement plus faible dans le cas des animaux du lot méthionine que dans celui des animaux du lot témoin, et cette différence est due surtout à une différence de teneurs des acides aminés considérés comme indispensables et semi-indispensables chez le monogastrique; la somme des concentrations sanguines de ces composés est diminuée d'environ I5 p. Ioo par l'addition de méthionine dans la ration. Cette baisse n'est pas observée également pour tous les acides aminés indispensables et semi-indispensables : la teneur en histidine est nettement 
augmentée et la teneur en lysine ne diminue pas, ce qui correspond à une augmentation de la proportion de cette dernière dans la somme des acides aminés " indispensables " et " semi-indispensables"; les teneurs en arginine et en méthionine ne sont que légèrement diminuées, alors que les teneurs en valine et en phénylalanine et, à un moindre degré, en isoleucine et leucine le sont plus que la moyenne. Les différences observées dans le cas des acides aminés non indispensables sont moins marquées : les teneurs en alanine et en citrulline sont diminuées dans une proportion supérieure à la moyenne, alors que la concentration de l'acide aspartique est augmentée ainsi que la proportion de glycine dans l'ensemble des acides aminés.

La somme des concentrations sanguines des acides aminés du cycle de l'urée est nettement plus faible dans le cas des animaux du lot méthionine que dans celui des animaux du lot témoin.

L'analyse des échantillons de sang prélevés chez les animaux du lot méthionine deux semaines après la suppression de la distribution de méthionine aux animaux met en évidence une augmentation de la teneur en acides aminés libres du sang, qui devient voisine de celle du sang des animaux témoins. Les teneurs en histidine et en lysine augmentent presque dans les mêmes proportions que celles des acides aminés indispensables, et se trouvent de ce fait nettement supérieures à celles qui ont été observées chez les animaux témoins. La somme des concentrations des acides aminés du cycle de l'urée augmente et atteint une valeur voisine de celle qui a été observée chez les animaux témoins.

\section{DISCUSSION}

Le taux butyreux et la quantité de matières grasses sécrétée dans le lait ont été significativement plus importants dans le lot méthionine que dans le lot témoin, ce qui est en accord avec leg résultats des essais effectués aux Etats-Unis avec le MHA (Polan et al., I970 ; PATTON et al., I970 b ; GRIEI et al., I968). En revanche, la quantité de lait sécrétée, l'augmentation de production laitière au début de la lactation, la concentration des protéines dans le lait et la quantité de protéines sécrétée n'ont pas été significativement plus importantes. On peut se demander si un apport plus faible de méthionine n'aurait pas provoqué une différence significative de la production laitière entre les 2 lots, à l'avantage du lot méthionine. En effet, $50 \mathrm{~g}$ de méthionine équivalent à $62 \mathrm{~g}$ de MHA, et PoLAN et al., (I970 a) ont montré qu'un apport de MHA supérieur à $45 \mathrm{~g}$ par jour était défavorable à une sécrétion maximale de lait; en fait, cette action défavorable semblait être la conséquence non pas d'une action dépressive directe du MHA sur la sécrétion du lait, mais plutôt d'un effet dépressif sur les quantités d'aliments ingérées. Or, dans notre essai, les quantités ingérées étaient les mêmes dans les 2 lots.

Pour les 7 semaines de lactation, les différences de poids vif entre les 2 lots d'animaux reflètent approximativement une différence de poids vif vide; en effet, les quantités d'aliments ingérées étant les mêmes dans les 2 lots, on peut penser que les poids des contenus digestifs étaient aussi les mêmes. Bien que dans le lot témoin, le poids vif des animaux ait augmenté dès le début de la lactation, il est probable que le poids vif vide ait diminué, au moins pendant les 2 ou 3 premières semaines : 
en effet, la teneur du plasma en acides gras libres était élevée et le bilan énergétique des animaux était négatif. Mais contrairement à ce qui s'est probablement produit dans le lot méthionine, l'augmentation du poids des contenus digestifs a été plus importante que la diminution du poids vif vide.

Il apparaît donc que c'est grâce à une mobilisation plus importante de leurs réserves corporelles que les vaches du lot méthionine ont pu sécréter dans le lait une quantité plus importante de matières grasses. C'est ce que semblent d'ailleurs confirmer la teneur plus élevée en acides gras libres plasmatiques (de façon non significative) et en corps cétoniques sanguins $(\mathrm{P}<0, \mathrm{IO})$, la proportion plus importante (de façon non significative) des acides gras longs du lait et le rapport

$$
\frac{\text { acétone }+ \text { acétoacétate }}{\beta \text {-hydroxybutyrate }}
$$

plus élevé dans le lot méthionine que dans le lot témoin. Ce mode d'action de la méthionine, probablement indirect, n'en exclut pas d'autres, en particulier une augmentation de la digestibilité de la ration, comme le suggèrent PoLAN et al. (I97o b) ou une activité de synthèse accrue de la glande mammaire, puisque non seulement la quantité des acides gras longs sécrétée provenant des lipides corporels a augmenté mais aussi celle des acides gras courts synthétisée. Mais la seule différence de poids vif à la $5^{\mathrm{e}}$ semaine de la lactation ( $\mathrm{I} 5 \mathrm{~kg}$ ) suffit à expliquer la différence de production laitière des animaux après 5 semaines de lactation ( $44 \mathrm{~kg}$ de lait à $4 \mathrm{p}$. Ioo de matières grasses).

La diminution de la concentration dans le sang des acides aminés indispensables, de ceux du cycle de l'urée et la légère augmentation de la proportion de la méthionine dans les acides aminés indispensables en présence de $50 \mathrm{~g}$ de méthionine supplémentaire dans la ration, et les variations en sens inverse après suppression de sa distribution, suggèrent que la méthionine était 1'acide aminé le plus limitant de l'organisme de l'animal et qu'une partie de la méthionine distribuée en supplément a été absorbée : l'apport supplémentaire de méthionine à l'animal hôte a pu s'effectuer d'au moins deux façons : d'une part, par une absorption directe à travers la paroi du rumen, ou par un passage du rumen à l'intestin d'une partie de la méthionine ingérée, sous forme libre ou liée mais non métabolisée ; d'autre part, par une augmentation de la quantité de protéines microbiennes synthétisée dans le rumen, principalement par les protozoaires ; cet accroissement de la quantité de protozoaires est suggéré par l'augmentation dans le sang des vaches du lot méthionine de la concentration de la lysine et de l'histidine, acides aminés dont les protozoaires sont plus riches que les bactéries (FAUCONNEAU et PION, I966) ; cela confirmerait les résultats obtenus par Patron et al. (I968, I970 $a$, I970 $c$ ).

Sachant que la méthionine participe à la synthèse des phospholipides et à la liaison des lipides et des protéines dans les lipoprotéines sériques (références citées par McCarthy et al., I968), peut-on penser que l'apport supplémentaire de méthionine a facilité la formation de lipoprotéines et ainsi permis une sécrétion plus importante de lipides dans le lait? Ou bien cela a-t-il été provoqué, et alors comment, par un meilleur équilibre des acides aminés obtenu grâce à 1'apport supplémentaire de méthionine? Il est aussi possible que la méthionine distribuée en supplément aux animaux ait agi autrement que par sa fonction d'acide aminé indispensable. Chez le poussin, Charkey (I959) a suggéré que la méthionine pourrait agir comme agent 
de couplage de réactions biochimiques, ou en augmentant la synthèse d'un agent de couplage, ou en interférant dans l'action de découplage de l'hormone thyroildienne.

Après 1a cessation de la distribution de méthionine, outre une augmentation de la concentration de la somme des acides aminés indispensables et de ceux du cycle de l'urée, et une diminution de la proportion de la méthionine dans les acides aminés indispensables, nous avons observé une augmentation de la concentration de la lysine et de l'histidine dans le sang, plus faible cependant que la moyenne des autres acides aminés. Les effets de la suppression de la distribution de la méthionine se manifesteraient donc plus rapidement chez l'animal hôte que chez les microorganismes du rumen.

Puisque, directement ou indirectement, 1a distribution de $50 \mathrm{~g}$ de méthionine a entraîné une production supplémentaire de matières grasses dans le lait, comment expliquer qu'à la $7^{\mathrm{e}}$ semaine de la lactation la production de matières grasses des animaux du lot méthionine ne soit pas devenue la même que celle des animaux du lot témoin? Le besoin en méthionine était probablement plus faible à la $7^{\mathbf{e}}$ semaine de la lactation qu'à la $5^{\mathbf{e}}$ semaine ; en effet, entre la $5^{\mathbf{e}}$ et la $7^{\mathbf{e}}$ semaine, les quantités de matières azotées et de matières grasses produites par jour dans le lait par les animaux du lot méthionine ont diminué respectivement de $40 \mathrm{~g}(4,6 \mathrm{p}$. 10o) et $72 \mathrm{~g}$ (7,5 p. 100), tandis que la quantité de matières azotées digestibles ingérée a augmenté de I47 g $(8,6$ p. I0o). Par ailleurs, il est vraisemblable que la durée d'enregistrement de la production des animaux après la cessation de la distribution de méthionine n'a pas été suffisamment longue.

Nos résultats permettent de penser que des vaches dont le niveau de production laitière est élevé peuvent être carencées en méthionine au début de la lactation. Pour des vaches qui ingéraient $2 \mathrm{I} \mathrm{kg}$ d'une ration constituée de foin de luzerne et de maïs, JACOBSON et al. (1970) ont calculé la quantité d'acides aminés que les animaux avaient ingérée et produite dans le lait. Pour chaque acide aminé, le rapport de la quantité ingérée à la quantité produite était supérieur à I, mais c'est pour la méthionine qu'il était le plus faible.

La quantité journalière de méthionine distribuée aux animaux $(50 \mathrm{~g})$ a été choisie d'après les résultats des expériences effectuées avec le MHA aux États-Unis. Or on sait que la méthionine est utilisée dans le rumen en proportion plus grande que le MHA, ce qui n'est peut-être pas un désavantage si elle stimule la synthèse des protéines microbiennes. Il serait donc nécessaire de comparer les productions de vaches recevant des quantités différentes de méthionine. Avec le régime que nous avons utilisé, la distribution de méthionine a entraîné la production d'un supplément de matières grasses dans le lait aux dépens des lipides corporels. Il serait intéressant d'étudier l'action de la méthionine distribuée en supplément de régimes qui tendent à orienter le métabolisme des lipides de la vache laitière vers la synthèse de matières grasses corporelles, comme les régimes à base d'ensilage de mais ou à proportion élevée d'aliments concentrés.

Avec différents régimes et des vaches dont les niveaux de production laitière seraient différents, il faudrait étudier le besoin en acides aminés, en particulier au début de la lactation; c'est en effet au cours de cette période que les risques de carence sont les plus grands, puisque les besoins de production sont maximum et les quantités d'aliments ingérées par les animaux minimum; il en résulte un faible 
apport protéique d'origine alimentaire et aussi d'origine microbienne car la synthèse de protéines par les microorganismes est liée de façon étroite à l'apport d'énergie.

Rę̧u pour publication en mai 1971.

\section{REMERCIEMENTS}

Cette étude a été réalisée avec le concours de l'A. E. C., Société de Chimie organique et biologique, et nous remercions le Service technique de cette entreprise pour sa participation.

\section{SUMMARY}

\section{EFFECT OF A SUPPLEMENT OF DL-METHIONINE FOR COWS AT THE START OF I,ACTATION ON PRODUCTION OF MILK AND COMPOSITION OF BLOOD}

The effect on milk production was studied of giving $5^{\circ} \mathrm{g}$ DL-methionine daily in the concentrate to cows at the start of lactation.

There were 2 groups of I I cows. The cows in one group were given the supplement of methionine from 2 weeks before until 5 weeks after calving. After stopping the methionine the cows remained in the experiment until the seventh week of lactation. The forage ration was of grass hay and silage to appetite and $3 . \mathrm{I} \mathrm{kg}$ beet. The amount of concentrate was increased from the beginning of lactation to meet the requirement of each cow towards the end of the first month of lactation

Individual intakes of feed, milk yield and composition and weights of the cows were recorded. At the end of the first week of lactation and during the third and seventh weeks blood was sampled. Blood sugar and ketone bodies and total lipids and free fatty acids in plasma were estimated

On the same dates, milk was sampled and the fatty acid composition of the mixture was estimate d. Blood samples were also taken during the fifth week of lactation from cows in both groups and during the seventh week from cows given methionine for estimation of the concentrations of free amino acids on mean samples for the groups.

The main results were as follows :

I. Intakes of the feeds were the same in both groups (table 2).

2. After 5 weeks of lactation yields of milk and of protein and protein content of the milk did not differ significantly between the 2 groups (table 2 ; fig. r). However, yield of fat and fat content of milk were significantly greater $(P<0.05)$ in the group given methionine than in the controls, by $82 \mathrm{~g}$ per day and $3.3 \mathrm{~g}$ per $\mathrm{kg}$, respectively.

3. Liveweight of the cows increased from the beginning of lactation in controls. It decreased for 3 weeks then increased slightly in the group given methionine (fig. I). Difference in liveweight between groups after 5 weeks of lactation was significant $(17.2 \mathrm{~kg} ; \mathrm{P}<0.05$, table 2).

4. Fatty acid composition of milk lipids did not differ significantly between the 2 groups (table 3). However, the proportion of long-chain fatty acids (C I $8+\mathrm{C} \mathrm{I} 8: \mathrm{I})$ tended to be greater in the group given methionine than in controls. The amount secreted of each class of fatty acids : short ( $\mathrm{C}_{4}$ to $\mathrm{C}_{\mathrm{I} 2}$ ), medium ( $\mathrm{C}_{\mathrm{I}} 6+\mathrm{C}_{\mathrm{I}} 6: \mathrm{I}$ ) and long (C I $8+\mathrm{C}_{\mathrm{I}} 8: \mathrm{I}$ ) was greater in the group given methionine than in the controls except for the short-chain fatty acids in the first week of lactation.

5. Concentation of ketone bodies in blood and the ratio of acetone + acetoacetic acid : $\beta$ hydroxybutyric acid were higher in the group given methionine than in the control group (table 4). The ame was true of free fatty acids in plasma, but the difference was not significant. At the fifth week of lactation the concentration of total free amino acids in blood was less in the group given methionine than in controls, mainly because of a reduction in concentration of the essential amino acids (table 5) ; concentration of amino acids of the urea cycle also was less. In contrast to the other essential amino acids, concentrations of methionine and lysine were not less, and 
that of histidine was even higher in the group given methionine than in controls.

It seems that methionine might act at the level of digestion by stimulating growth of rumen micro-organisms, particularly the protozoa, at the metabolic level by increasing the mobilisation of lipid bodies and also, perhaps, at the level of the mammary glands by increasing synthesis.

\section{RÉFÉRENCES BIBLIOGRAPHIQUES}

Breirem K., 1954. Die nettoenergie als grundlage der bewertung der futermittel in : NehriNG K., Hundert jahre Mockern. Die Bewertung der Futterstoffe und andere Probleme der Tiernährung. Berlin, Deutsche Akad. der Landwirtschaftswissenchaften, t. II, 97-108.

Broderick G. A., Kowalczyck T., Satter L. D., 1970. Milk production response to supplementation with encapsulated methionine per os or casein per abomasum. J. Dairy Sci., 58, I714-172I.

Burgos A., Olson H. H., 1970. Effects of $40 \mathrm{~g}$ of methionine hydroxy analog on yield and composition of milk. J. Dairy Sci., 53, 647 (Abstr.).

Chander P. T., Polan C. E., Shaw D. R., r97o. Methionine hydroxy analog and lipid metabolism in the lactating cows. J. Dairy Sci., 58, 376 (Abstr).

Charkey L. W., 1959. Methionine, iodocasein and oxygen consumption of chicks. J. Nutr., 69, 295-300.

DEcaEN C., ADDA J., I966. Évolution de la sécrétion des acides gras des glycérides du lait de vache au cours de la lactation. XVII Congr. Internat. Laiterie, 1, I6I-I7I.

Decaen C., Journet M., Poutous M., I97o. Évolution de la production laitière de la vache au cours des deux premiers mois de la lactation. İ. Description graphique de l'évolution journaliere de la quantité de lait sécrétée, du taux butyreux et de la quantité de matières grasses sécrétée. Ann. Zootech., 19, r9I203.

Dole P., Meinertz H., I960. Determination of long-chain fatty acids in plasma and tissues. $J$. Biol. Chem., 285, 2595-2599.

Fauconneau G., Pron R., x966. Amino acid composition of proteins of some fodder plants. Pric. IX Internat. Grassland Congr., Vol. 1, 779-781.

Fisher L. J., ERfLE J. D., rg7o. Effect of the intravenous infusion of either glucose or L-methionine on lactating cows exhibiting symptoms of ketosis. J. Dairy Sci., 53, 664 (Abstr.).

Folch J., Lees M., Sloane-Stanley G. H., 1957. A simple method for the isolation and purification of total lipids from animal tissues. $J$. Biol. Chem., 228, 497-509.

Griel Jr. L. C., Patton R. A., McCarthy R. D., Chandler P. T., 1968. Milk production response to feeding methionine hydroxy analog to lactating cows. J. Dairy Sci., 51, I866-1867.

Hugget A. S. G., Nixon D. A., I957. Enzymic determination of blood glucose. Biochem. J., 66. I2p.

Jacobson D. R., VAN Horn H. H., Sniffen. C. I., r97o. Lactating ruminants. Fed. Proc., 29, 35-40.

Leroy A. M., I949. Ve Congr. Intern. de Zootechnie. Paris. Vol I.

McCarty R. D., Porter G. A., Griel Jr. L. C., I968. Bovine ketosis and depressed fat test in milk : a problem of methionine metabolism and serum protein aberration. J. Dairy Sci., 51, 459-462.

Patton R. A., McCarthy R. D., Griel Jr L. C., 1968. Lipid synthesis by rumen microorganisms. I. Stimulation by methionine in vitro. J. Dairy Sci., 51, I3ro-r3II.

Patton R. A., McCarthy R. D., Griel Jr. L. C., r97o a. Lipid synthesis by rumen microorganisms. II. Further characterisation of the effects of methionine. J. Dairy Sci., 58, 460-465.

Patton R. A., Mc Carthy R. D., Griel Jr. L. C., i97o $b$. Observations on rumen fluid, blood serum, and milk lipids of cows fed methionine hydroxy analog. J. Dairy Sci., 53, 776-780.

Patton R. A., Mc Carthy R. D., Keske L. G., Griel Jr. L. C., Baumgardt B. R., i97o $c$. Effect of feeding methionine hydroxy analog on the concentration of protozoa in the rumen of sheep. $J$. Dairy Sci., 53, 933-935.

Pawlak M., Pion R., Ig68. Influence de la supplémentation des protéines du blé par des doses croissantes de lysine sur la teneur en acides aminés libres du sang et du muscle du rat en croissance. $A n n$. Biol. anim. Bioch. Biophys., 8, $517-530$.

Polan C. E., Chandler P. T., Mrller C. N., r97oa. Methionine hydroxy analog : varying levels for lactating cows. J. Dairy Sci., 58, 607-6ro.

Polan C. E., Chandier P. T., Miller C. N., I970 b. Methionine analog effect on ruminant digestion. J. Anim. Sci., 31, 25 I (Abstr.).

Procos J., 196I. Modification of the spectrophotometric determination of ketone bodies on blood enabling the total recovery of $\beta$-hydroxybutyric acid. Clin. Chem., 7, 97-ro6.

Teichman R., Caruolo E. V., Mochrie R. D., I969. Milk production and composition responses to intravenous infusion of L-methionine. J.Dairy Sci., 52, 942 (Abstr.).

Williams L. R., Martz F. A., Hilderbrand E. S., r97o. Feeding encapsulated methionine supplement to lactating cows. J. Dairy Sci., 53, I709-I7I3 